\title{
Intergroup Joint Scheduling for Mitigating Asymmetric Uplink Interference in Self-Organizing Virtual Cell Networks
}

\author{
Ohyun Jo, ${ }^{1}$ Gyung-Ho Hwang, ${ }^{2}$ and Dong-Ho Cho ${ }^{3}$ \\ ${ }^{1}$ Electronics and Telecommunications Research Institute (ETRI), Daejeon, Republic of Korea \\ ${ }^{2}$ Department of Computer Engineering, Hanbat National University, Daejeon, Republic of Korea \\ ${ }^{3}$ School of Electrical Engineering, Korea Advanced Institute of Science and Technology (KAIST), Daejeon, Republic of Korea \\ Correspondence should be addressed to Gyung-Ho Hwang; gabriel@hanbat.ac.kr
}

Received 26 August 2016; Accepted 18 October 2016

Academic Editor: Seok-Chul Kwon

Copyright (C) 2016 Ohyun Jo et al. This is an open access article distributed under the Creative Commons Attribution License, which permits unrestricted use, distribution, and reproduction in any medium, provided the original work is properly cited.

\begin{abstract}
We introduce the concept of self-organizing VCN (virtual cell network). Here self-organizing VCN topology for efficient operation will be configured, and the functions of the each element will be defined. Also, the operation scenarios of VCN will be described. Then, we propose an efficient scheduling algorithm that considers the asymmetry of interference between downlink and uplink to mitigate intercell interference with little computing overhead. The basic concept is to construct scheduling groups that consist of several users. Each user in a scheduling group is affiliated with a different cell. Then, the intercell groups are managed efficiently in the proposed VCNs. There is no need for the exchange of a lot of information among base stations to schedule the users over the entire network.
\end{abstract}

\section{Introduction}

Interference needs to be managed strictly, because, in recent years, the portion of the cell area that is overlapping with other cells has been increasing as cell sizes have been getting smaller. This increase of overlapped region means that the effect of intercell interference is increased, due to the increased cell edge area. Users located in the edge area of a cell are seriously affected by the increased interference. This is the main reason for the degradation of system performance. Conventional algorithms that have been deployed to tackle this problem are usually restricted by a single-cell optimization [1].

However, the news is not all bad. A new network topology that offers more coordination between antennas has been proposed for next-generation systems. This system environment is considered in recent communication specifications for improving system performance [2]. For instance, the third-generation partnership project (3GPP), which is the representative association for system standardization, introduces coordinated multipoint transmission/reception (CoMP) for joint processing among multiple base stations [3]. Each base station is connected to a coordinator, which controls cooperation among the base stations by collecting and passing of information. Intra-e-nodeB of the 3GPP is a further example of coordinated networks [4]. In an attempt to improve system performance further, more coordinated network topology has been proposed for 5th-generation systems $[5,6]$. This system environment has featured prominently in recent communication specifications for improving system performance. For instance, each base station is connected to a coordinator, which controls cooperation. Some information for cooperation is exchanged or collected by the coordinator.

Motivated by the research described above, we herein propose an innovative network architecture and a groupscheduling algorithm for efficient resource management. Traditional cellular system, where each base station independently manages all processing within the coverage, has many obstacles in view of drastically improving the system performance due to interference. Particularly, when cell size is reduced, cell edge area increases and handoff occurs more frequently. Even though partial cooperation between base stations somehow helps this limitation, it also causes much overhead due to information exchange. Motivated by the above-mentioned recent research, we herein propose a coordinated VCN topology in which intercell interference is mitigated. The particular means of coordination that we 


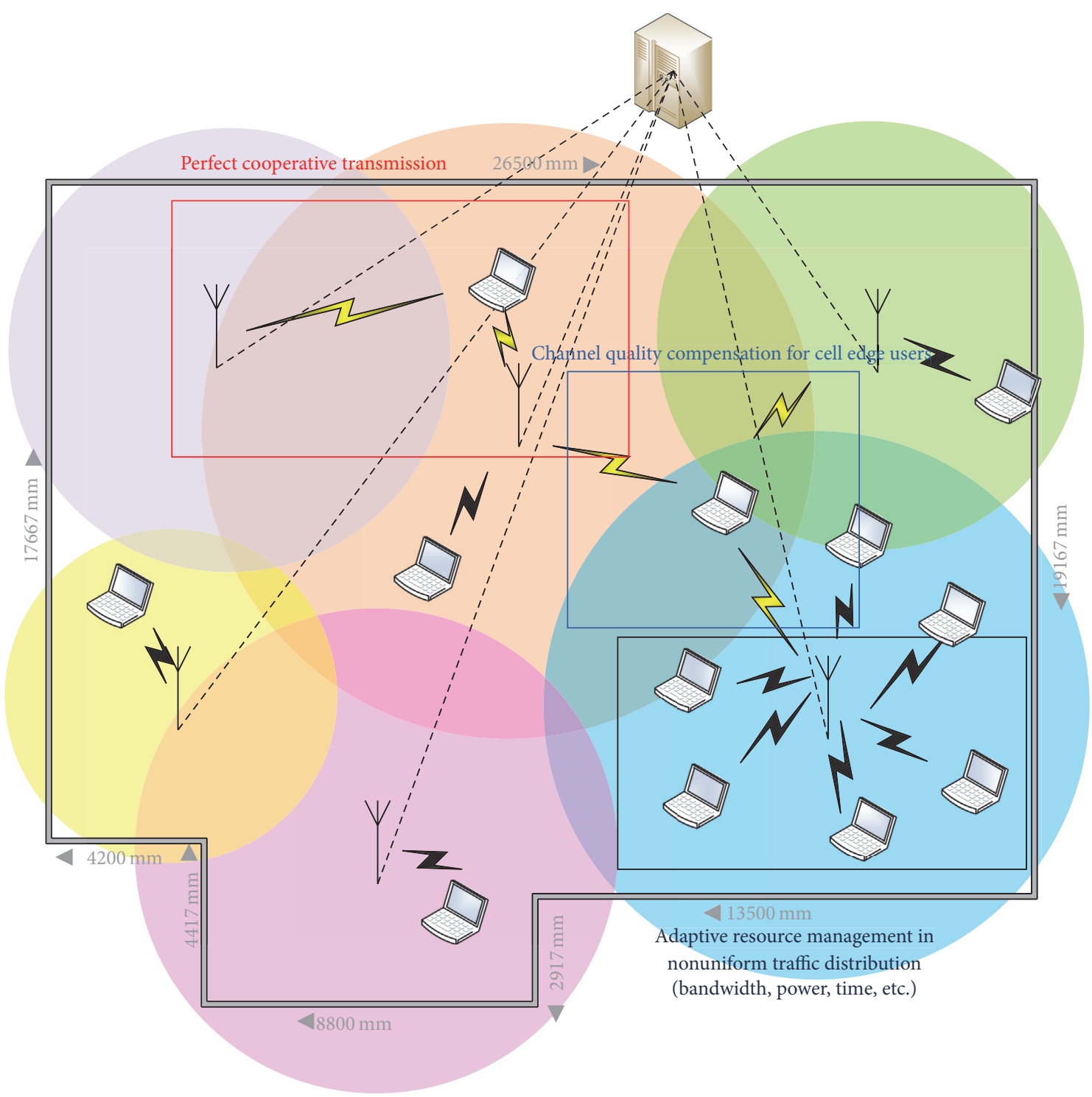

FIGURE 1: System configuration for VCN.

propose enable us to implement the joint scheduling of multiple base stations in the network efficiently, with low computational complexity. The basic idea of VCN is totally self-organizing network architecture to avoid signal overhead among base stations and make feasible full cooperation. Each cell which is represented as RAU (Radio Access Unit) in VCN only has minimal mandatory functions for working and is actually managed by one master unit so-called CU (Central Unit) in the proposed network topology. Moreover, multiple cells can be dynamically merged into one virtual cell which is also managed by CU. So, each cell corresponding to an RAU is not a real cell. This is why we call the new network topology the virtual cell network. The new network topology manages a new resource domain.

\section{VCN System Configuration}

The basic idea of VCN is totally centralized architecture to avoid signal overhead among base stations and make full cooperation feasible. Each cell which is represented as RAU in VCN only has minimal mandatory functions for working and is actually managed by one master unit so-called CU in the proposed network topology.

New network architecture for VCN is given in Figure 1. VCN consists of two main components which are CU and RAU. CU has multiple high-level BS controllers and it is the core component in VCN. RAU has a low-level BS controller. And central controller in CU manages all BS controllers in the network. In order for an RAU to build up an independent single-cell, the RAU should have a connection to the highlevel BS controller and the central controller. In the case that multiple RAUs are merged into one virtual cell, multiple RAUs are connected to one high-level BS controller and the central controller. The function set of high-level BS controller and low-level controller can be defined in two ways. The first case is that high-level BS controller has function set of RRC, $\mathrm{MAC}$, and PHY, and low-level BS controller has function set 


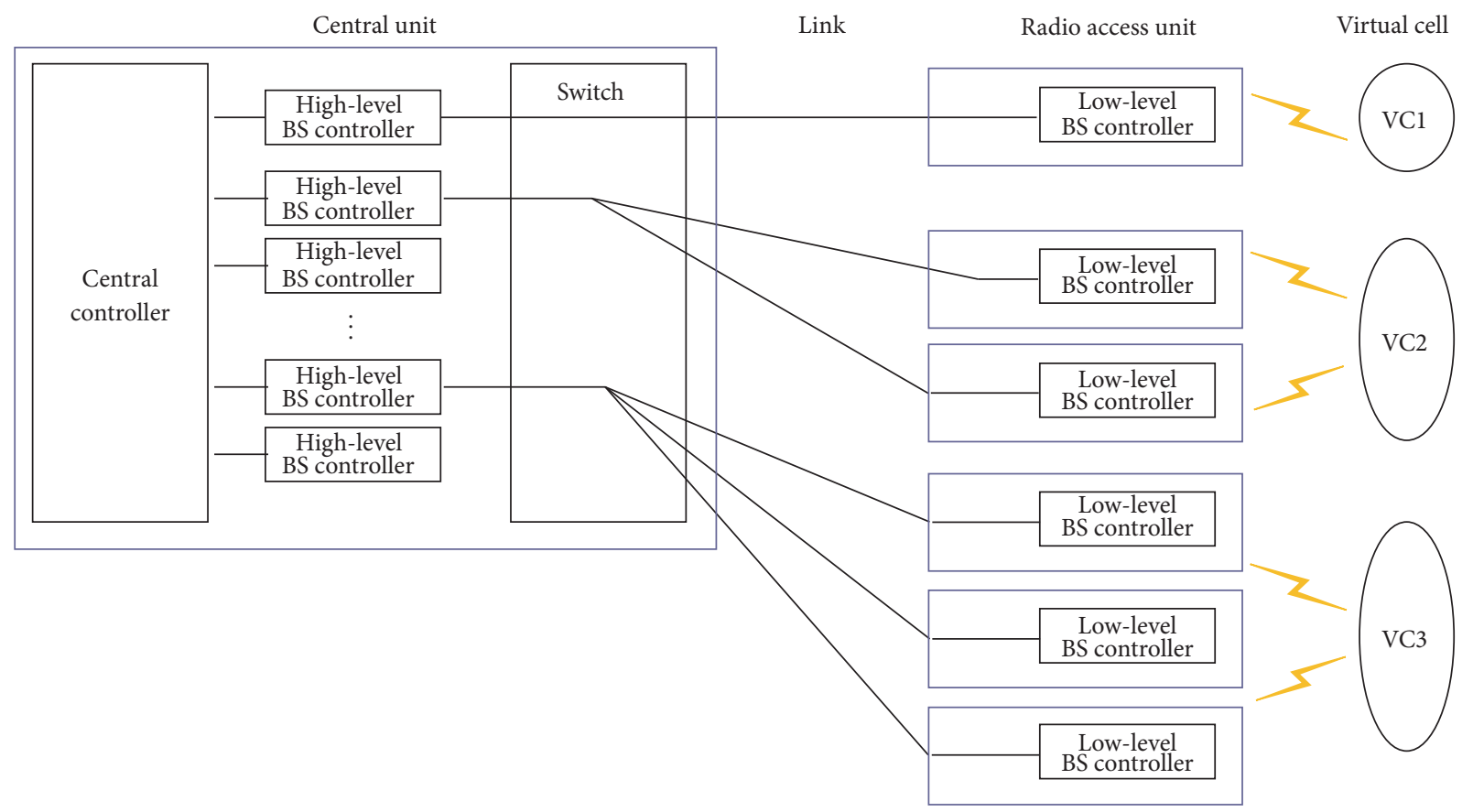

FIGURE 2: Basic conceptual diagram for VCN.

of RF and antenna. In this case, baseband signal is transmitted in the link between CU and RAU. The second case is the high-level BS controller that has function set of RRC, MA, PHY, and RF, and low-level BS controller has only function set of antenna. In this case, RF signal is transmitted in the link between CU and RAU.

Figure 2 shows the basic conceptual diagram for VCN. CU contains a switch module which carries out mapping function between high-level controller in CU and low-level controller in RAU. The connection is essential to make a virtual cell. To build up a cell, the functionalities of both controllers should be combined. If only one RAU is mapped to a high-level controller, the virtual cell contains only one antenna. In this case, the virtual cell can be considered as an independent normal cell which is just managed by CU. On the other hand, if multiple RAUs are connected to a high-level controller in CU, the virtual cell has multiple antennal. Therefore, the virtual cell can dynamically change the configuration according to the system environment. This is the main characteristic.

Figure 3 shows the functionality of CU. The backhaul interface is a submodule for the communication toward the outer networks, such as wired/wireless network. It is also useful for the cooperation between VCNs. Virtual cell database manages the overall information for the base station and terminals which are working in virtual cell mode. It contains channel information and traffic information and so on. Virtual cell manager carries out functions for managing virtual cell. It performs scheduling, resource allocation, and so on. User mobility manager manages user mobility in a virtual cell. In a virtual cell, new handover scenarios happen, because the cell structure is different from conventional communication systems. Users can move from VCN to
VCN (inter-VCN handover) and also can move to another antennal coverage area in a same virtual cell area (intra-VCN handover). Therefore, the complicated procedure should be carefully managed by this submodule. Intercell interference coordinator operates for the interference management in a virtual cell. The interference model is more complex in a virtual cell. Interference can be generated by neighbor antenna, neighbor VCN, or outer cellular network (in the case of overlaid network structure). In a virtual cell, careful interference management is required for avoiding performance degradation. Therefore, this submodule performs the important roles. Traffic QoS manager contains traffic information of users in a virtual cell. In a virtual cell, all kinds of traffic adaptive algorithm can be implemented based on sharing of traffic information. Finally, BS interface provides the connection between central controller and high-level BS controller in CU.

More advanced and efficient system configuration can be considered for improving the system performance. In this case, RAUs also should be more improved than the basic configuration to perform smarter operations. In this configuration, hierarchical resource management is possible. That makes it possible to increase the system efficiency. The system configuration is shown in Figure 4.

In this configuration, some smarter RAUs contain enhanced low-level BS controller. The RAU which has enhanced low-level BS controller can behave just like conventional pico cell or femto cell. The information of the cell is independently managed by the RAD by itself without any aid of CU. If an individual antenna builds up an independent cell, the enhanced low-level BS controller is activated. In this case, the RAU is not connected to high-level BS controller. Instead, it is directly connected to the central controller for enabling 


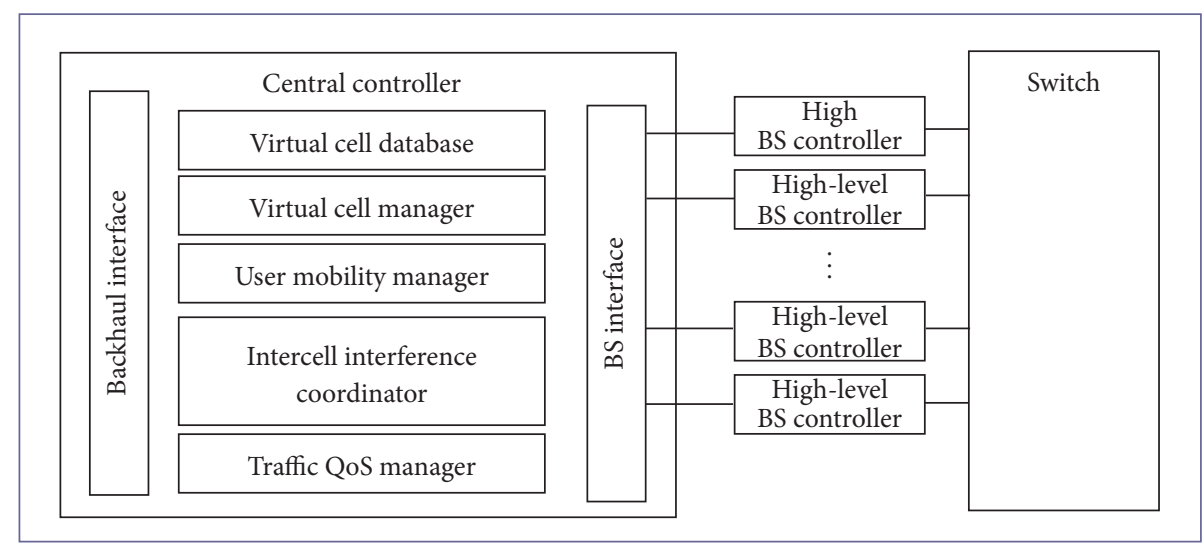

FIgURe 3: CU (Central Unit) functionality.

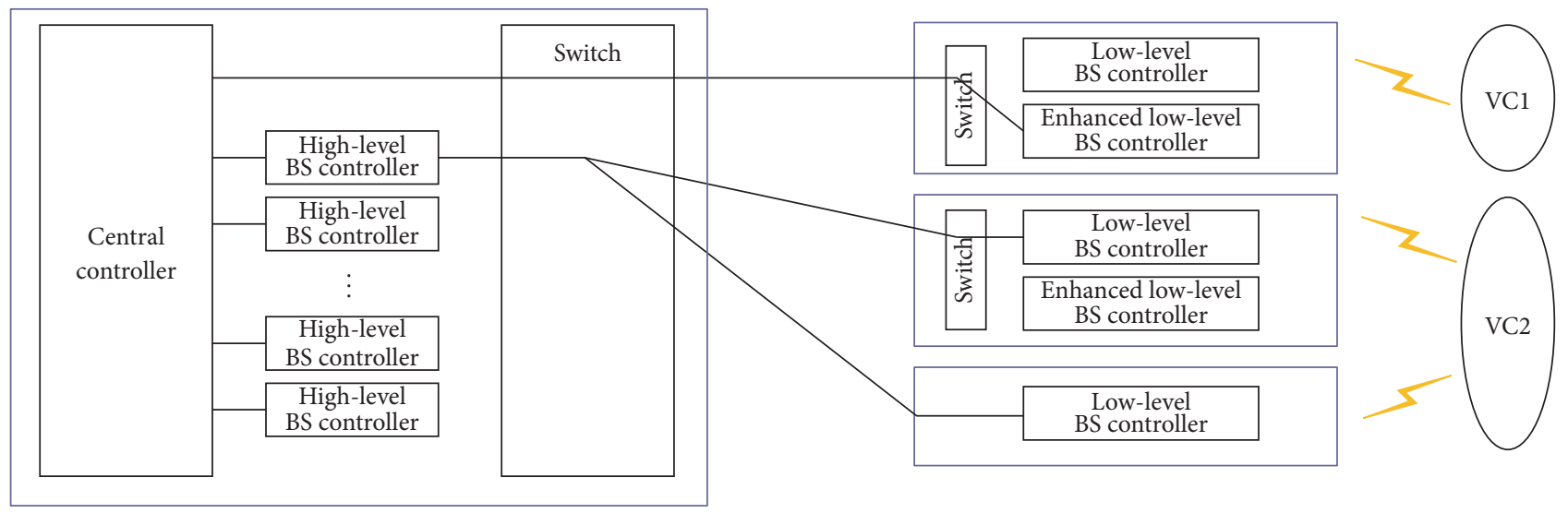

Figure 4: Conceptual diagram for advanced VCN.

network management. When the RAU builds up a virtual cell with other RAU, the enhanced low-level BS controller is not activated and the low-level BS controller is connected to the high-level BS controller in CU. For the feasibility of this new configuration, RAU needs to have a switch module which can configure the mode of RAU. Also, the RAUs with enhanced low-level BS controller can provide backward compatibility with the conventional pico cell base stations or femto cell base stations.

RAUs which have enhanced low-level BS controller can build up a virtual cell by itself. In this case, system configuration is shown in Figure 5.

In this configuration, the functions of high-level BS controller are replaced with enhanced low-level BS controller. Also, the RAU is directly connected to central controller not to the high-level BS controller. In the case that any kind of cooperation and management is not required, the Master RAU manages the virtual cell totally independently without any aid of Central Unit.

When the network size is very huge, sometimes only one $\mathrm{CU}$ is not capable to manage all the network. In this case, each CU forms a virtual cell network independently, and the several virtual cell networks can cooperate through an improved system configuration as shown in Figure 6.
Figure 7 shows the system configuration in the case that some RAUs independently build up a virtual cell without CU. Here, the head RAU which replaces the functions of $\mathrm{CU}$ is called Master RAU.

For enabling the virtual cell system configuration, mobile terminals also should be improved. Mobile terminals in VCN can request to build up or break up a virtual cell in some cases, considering channel quality, QoS, RAU status, cell shape, and so on. The RAU needs to request some information from mobile terminals to decide on the network topology. When the network topology is changed, the mobile terminals should be notified with this change from RAU for adaptation. For this, mobile terminals can receive the information and configure the functionality. Therefore, some submodules for performing those operations are required at mobile terminals as shown in Figure 8. Also, signaling procedure is described in Figure 9.

\section{Operation Scenario}

When the new network topology is applied in real communication system, abundant advantages can be obtained as shown in Figure 1. For example, unintended resource waste can be reduced in nonuniform traffic distribution environments. 


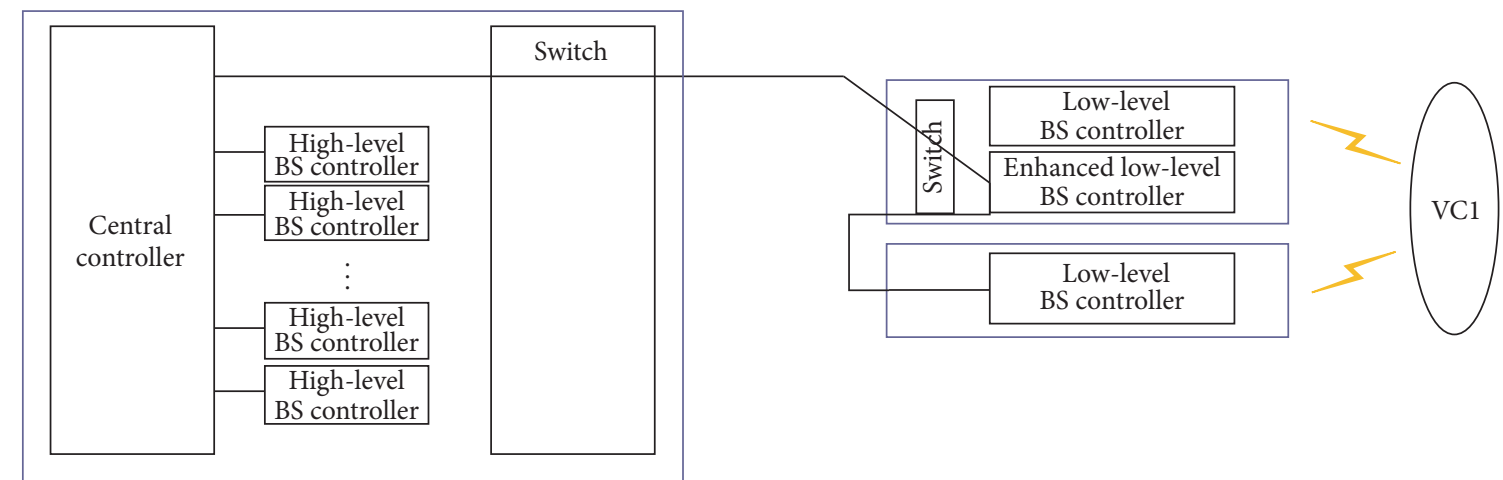

FIGURE 5: Conceptual diagram for self-organizing VCN.

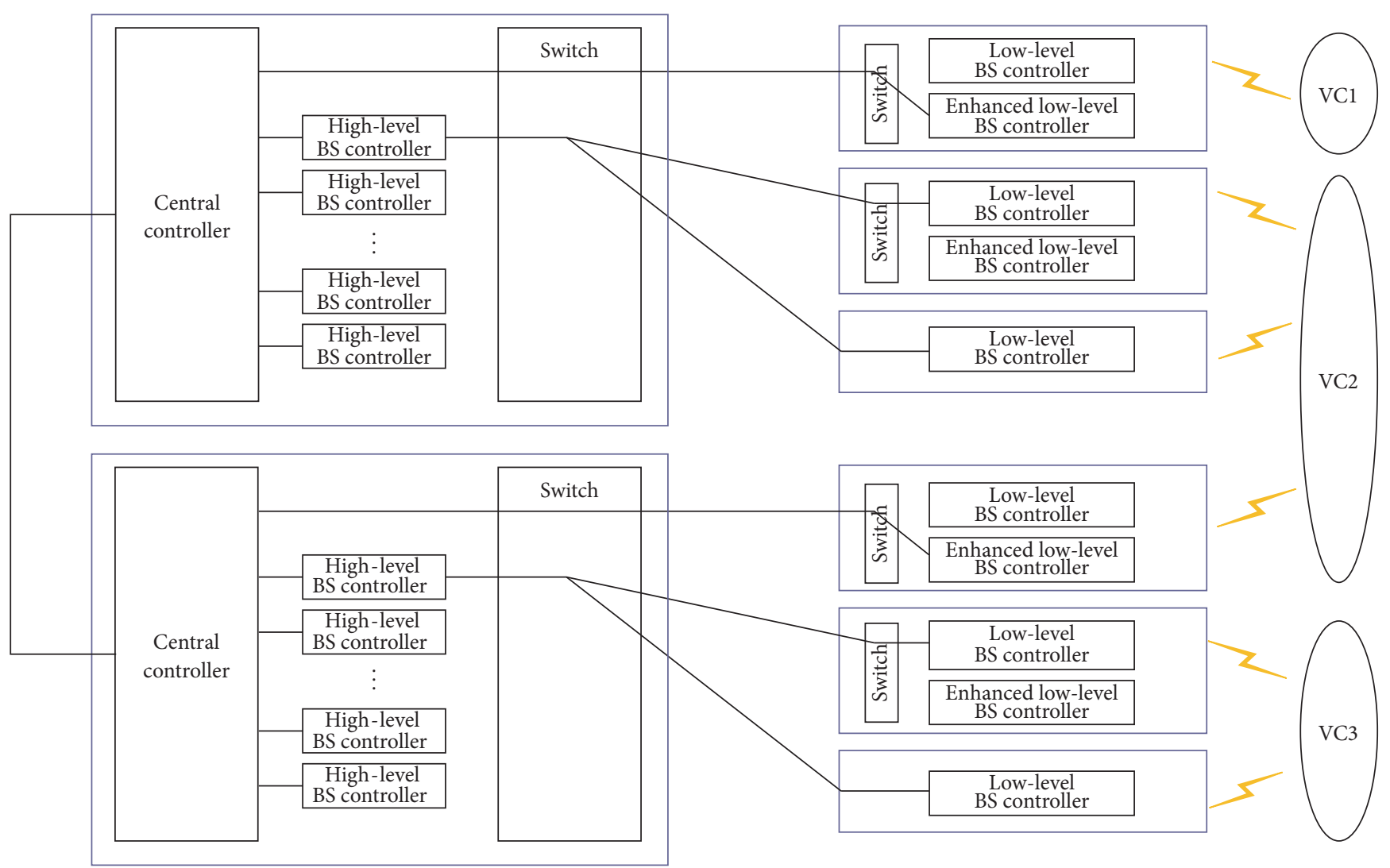

FIGURE 6: CU-concatenated VCN.

Also, full cooperation can increase the spectral efficiency, especially in the edge of the cell coverage. Scheduling and resource management also can be more efficient based on the information of traffic distribution, channel quality, and user mobility in all network.

Based on various network status, for example, traffic distribution, user priority, mobility, and so forth, VCN can dynamically change the form of a virtual cell. This is the basic and typical operation of VCN. Pretty much benefit is attained from this characteristic. If there is no user in a neighbor cell, the neighbor cell can be totally merged. In this case, the two antennas are managed by a common high-level BS controller; then it acts like a distributed MIMO system. In other words, it forms a virtual cell. If there is an idle resource in a neighbor cell, the neighbor cell can be partially merged. In this case, the resource of the partially merged cell is managed hierarchically. In the VCN frame structure, some part can be managed by low-level BS controller independently, and the other part can be separately managed by high-level BS controller in CU for cooperation. Finally, if there is no idle resource in a neighbor cell, each cell operates separately. One user associates with only one antenna in this case. 


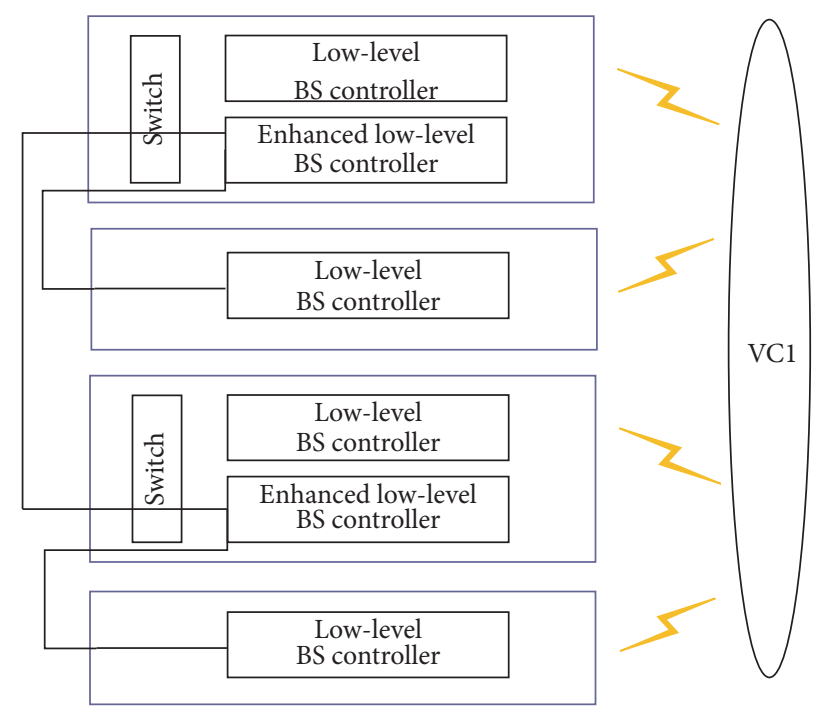

Figure 7: RAU-concatenated VCN.

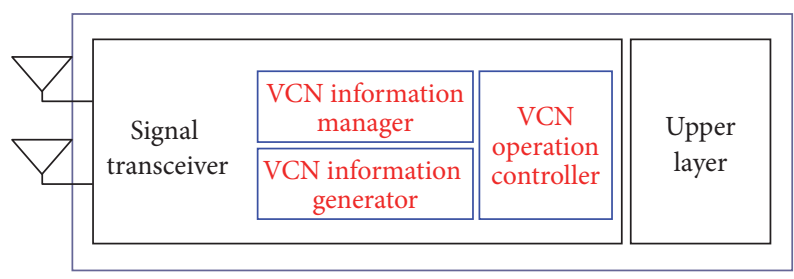

FIGURE 8: Conceptual diagram of mobile terminal.

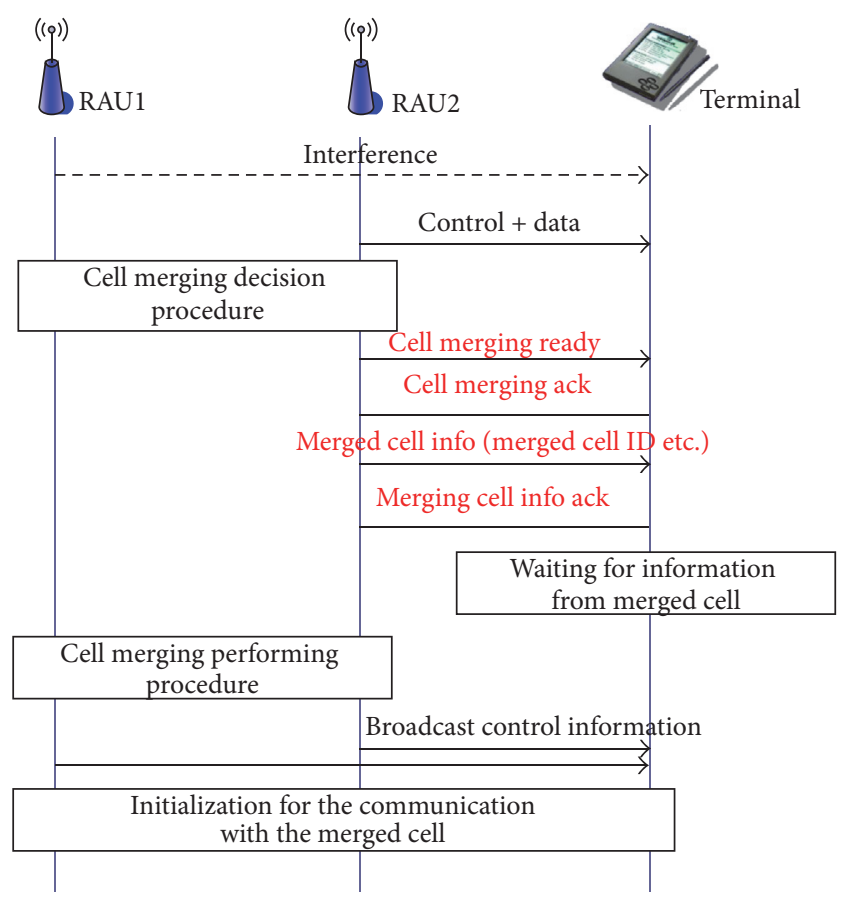

FIGURE 9: Signaling procedure for building up a virtual cell.

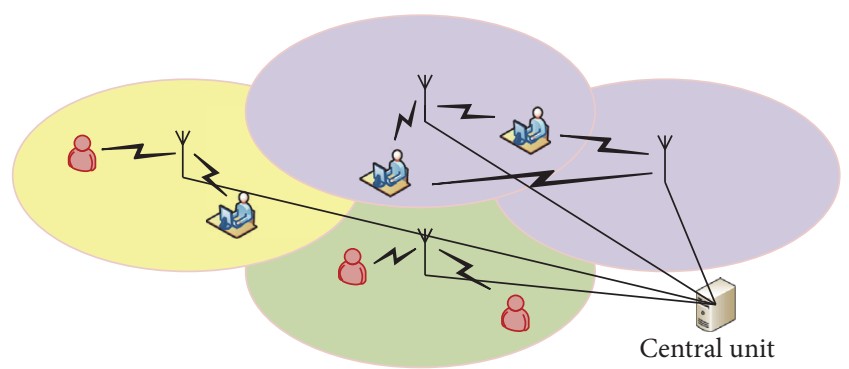

FIGURE 10: Complete cell merge scenario in VCN.

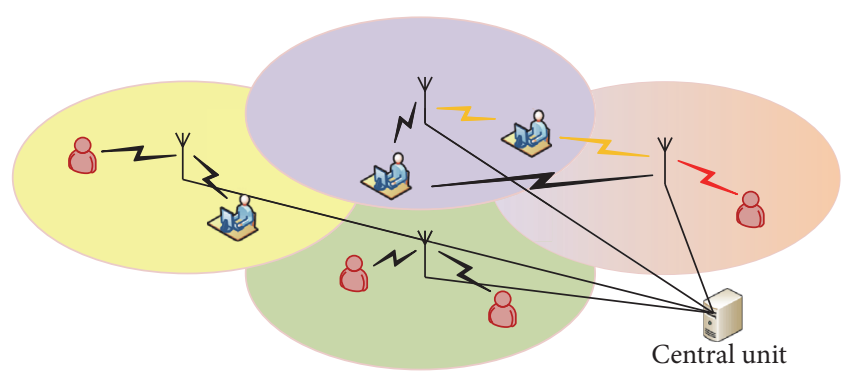

FIGURE 11: Partial cell merge scenario in VCN.

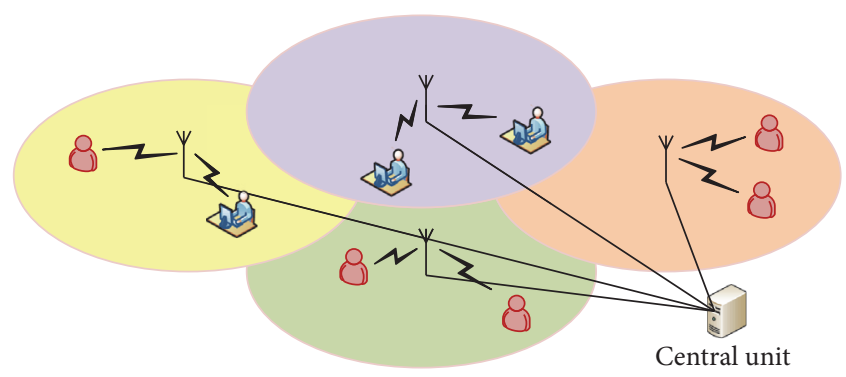

FIGURE 12: Cell separation scenario in VCN.

However, each cell can be managed by a common high-level BS controller for interference mitigation. These operations are shown in Figures 10, 11, and 12.

\section{Asymmetry Interference Problem}

There exists an asymmetric characteristic in interference between uplink and downlink. Mobile users in communication systems usually report the Channel Quality Index (CQI) based on the pilot signal from base stations. The pilot signal is a predefined signal pattern that enables mobile users to recognize the base stations. The base stations periodically transmit the pilot signal by using a predefined resource. Through this operation, mobile users can recognize the precise state of the channel and measure the Signal to Interference and Noise Ratio (SINR) in downlink. The situation for uplink is not so helpful. In this case, it is not easy for the BSs to determine the precise state of channels and measure the SINR, because they cannot recognize all the signal patterns that identify users of 


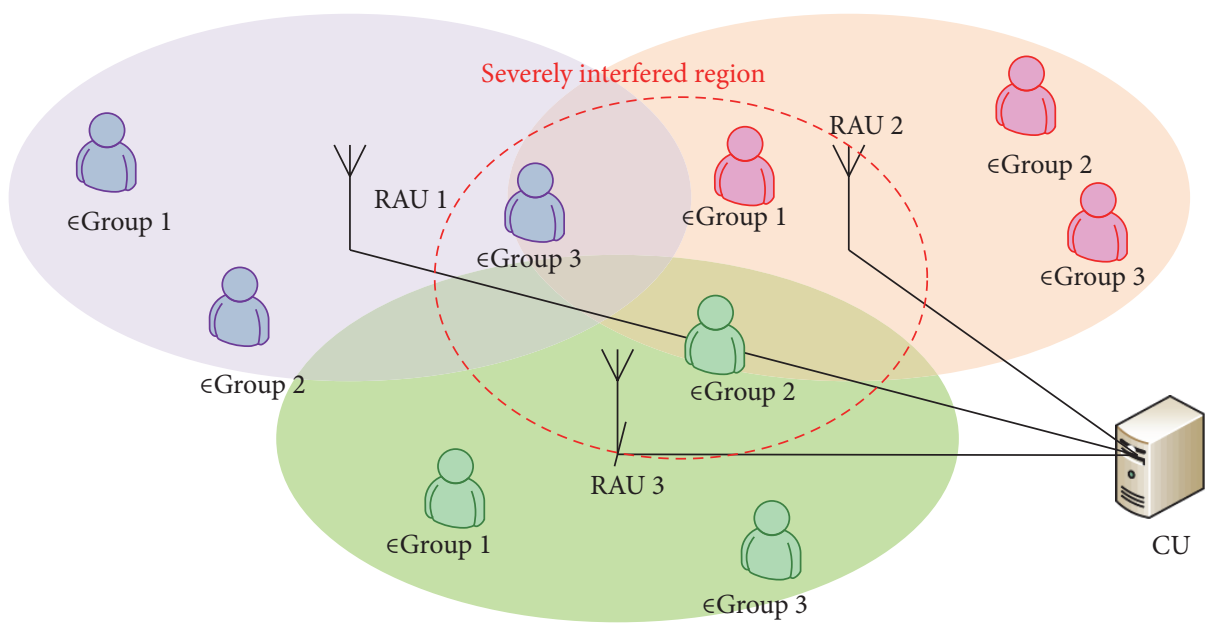

FIGURE 13: System model and the basic idea of the proposed intergroup joint scheduling algorithm.

neighbor cells. Moreover, mobile users may change location, unlike base stations, which are deployed in a fixed position. Consequently, uplink interference model is hard to predict. Therefore, it is much more difficult to manage interference in the uplink than in the downlink case. In most communication systems, the uplink channel quality is assumed to be the same as the downlink channel quality. Consequently, uplink scheduling is performed on the basis of the information about the channel quality that is obtained from CQI reports sent by mobile terminals. However, the CQI reports do not provide information that is correct for uplink. In some cases, even though the downlink SINR is very good, the uplink channel quality may, depending on the position of the mobile users, be pretty poor because of the asymmetry in interference. However, using the benefits of coordinated network topology, we can derive more accurate information about the uplink channel, thereby improving system performance.

\section{Intergroup Joint Scheduling}

5.1. Basic Idea. In VCNs, RAUs are able to cooperate through a CU, as shown in Figure 13. Here, we propose another approach for uplink scheduling using an intelligent network topology. The proposed approach, which we call intergroup joint scheduling, builds up uplink scheduling groups composed of multiple users who may be located in different cells. The members of a particular scheduling group are allocated to the same resource region by the CU. When the members of a scheduling group are allocated to the same resources, they are assumed to be hardly correlated with each other for the purposes of mitigating intercell interference. Figure 13 illustrates the basic idea of the intergroup scheduling.

5.2. Conditional SINR-Based Grouping. For the proposed uplink intergroup scheduling algorithm, we first define an exclusive cluster. The users in an exclusive cluster have low SINR in the long term in their channel. Usually, some of the users who have low SINR are located in the area that overlaps with other cells. In this area, the received signal strength from other neighbor cells is quite high. However, that means the users in the edge of the cell can also interfere with the uplink signal transmission of the neighbor cell. Most of the members of an exclusive cluster are located in the edge of the cell. Therefore, these severe interferers in the cluster should not share resources. Then, we build up a scheduling group for a user in the exclusive cluster based on conditional SINR.

Step 1. The CU collects information from users in VCNs to make an exclusive cluster. The information collected by the $\mathrm{CU}$ contains the signal strength of users from neighbor RAU cells. The CU makes an exclusive cluster from those users who satisfy the following condition:

$$
P_{r x}<\alpha \cdot \sum P_{\text {inter }},
$$

where $P_{r x}$ is the received power from the corresponding RAU of a user, $\sum P_{\text {inter }}$ is the sum of the received power of neighbor RAUs, and $\alpha$ is a scaling factor related to the bound of the exclusive cluster area. If $s$ users satisfy the above condition, $P_{\mathrm{ec}}$ is defined as a set composed of $s$ users who are in the exclusive cluster and $P_{\text {nec }}$ is defined as a set composed of $N-s$ users who are not in the exclusive cluster. Here, $u$ represents a user in $P_{\mathrm{ec}}, \psi$ represents a user in $P_{\mathrm{nec}}$, and $N$ is the total number of users in the network.

$$
\begin{aligned}
P_{\mathrm{ec}} & =\left\{u_{1}, \ldots, u_{s}\right\} \\
P_{\text {nec }} & =\left\{\psi_{1}, \ldots, \psi_{N-s}\right\} .
\end{aligned}
$$

Step 2. In the second step, we find a dominant interferer of each $u$ in $P_{\text {ec }}$. Here, we use the conditional SINR [7].

The conditional SINRs of subchannel $n$ for user $m$ in base station $\pi(m)$, that is, $\gamma_{m, n \mid \Psi=\{\}}^{\pi(m)}, \gamma_{m, n \mid \Psi=\left\{\psi_{1}\right\}}^{\pi(m)}, \gamma_{m, n \mid \Psi=\left\{\psi_{2}\right\}}^{\pi(m)}, \ldots$, $\gamma_{m, n \mid \Psi=\left\{\psi_{N-s}\right\}}^{\pi(m)}$, which correspond to restrictions of none, $\psi_{1}, \psi_{2}, \ldots, \psi_{N-s}$, respectively, are calculated from the SINR expression. $\pi(m)$ denotes the corresponding RAU of user $m$

$$
\gamma_{m, n}^{\pi(m)}=\frac{P_{c} H_{m, n}^{\pi(m)}}{P_{c} \sum_{k \neq m} H_{k, n}^{\pi(m)} I_{k, n}+P_{N}},
$$


where $P_{c}$ is the transmit power supplied on each subchannel derived from equal power distribution, $P_{N}$ is the thermal noise power over the subchannel bandwidth, and $H_{m, n}^{\pi(m)}$ and $H_{k(k \neq m), n}^{\pi(m)}$ are the link gains to the registered RAU of user $m$ and the interferer $k$, respectively. The indicators $I_{k, n}$ take the value 1 or 0 depending on whether or not the interferer $k$ is scheduled in subchannel $n$. If user $k$ is restricted in subchannel $n, I_{k, n}$ will be definitely 0 .

Using the definition of conditional SINR, we calculate the conditional SINRs in the case that each user in $P_{\text {nec }}$ is restricted. Then, to build up a scheduling group, we determine whether or not each conditional SINR satisfies the following condition.

$$
c_{m, n \mid \Psi=\{\}}^{\pi(m)}>c_{m, n \mid \Psi=\left\{\psi_{i}\right\}}^{\pi(m)}+c_{\psi_{i}, n \mid \Psi=\{m\}}^{\pi\left(\psi_{i}\right)} \quad \text { for } i=1, \ldots, N-s .
$$

Each term of the above equation is defined by a Shannon's equation as follows. Here, $B$ is the bandwidth of subchannel $n$.

$$
\begin{gathered}
c_{m, n \mid \Psi=\{\}}^{\pi(m)}=B \cdot \log _{2}\left(1+\gamma_{m, n \mid \Psi=\{\}}^{\pi(m)}\right) \\
c_{m, n \mid \Psi=\left\{\psi_{i}\right\}}^{\pi(m)}=B \cdot \log _{2}\left(1+\gamma_{m, n \mid \Psi=\left\{\psi_{i}\right\}}^{\pi(m)}\right) \\
c_{\psi_{i}, n \mid \Psi=\{m\}}^{\pi\left(\psi_{i}\right)}=B \cdot \log _{2}\left(1+\gamma_{\psi_{i}, n \mid \Psi=\{m\}}^{\pi\left(\psi_{i}\right)}\right) .
\end{gathered}
$$

Using this condition as a basis, we define and identify preferred candidates of each member in $P_{\mathrm{ec}}$ for building a scheduling group. The preferred candidates are determined for every member in $P_{\mathrm{ec}}$ as follows. Then, $S_{m}$, which is a set of preferred candidates of user $u_{m}$ in $P_{\text {ec }}$, can be expressed as

$$
\begin{aligned}
S_{m}=\left\{\psi_{k} c_{m, n \mid \Psi=\{\}}^{\pi(m)}>c_{m, n \mid \Psi=\left\{\psi_{k}\right\}}^{\pi(m)}+c_{\psi_{k}, n \mid \Psi=\{m\}}^{\pi\left(\psi_{i}\right)}\right\} & \\
& \text { for } m=1, \ldots, s .
\end{aligned}
$$

Step 3. Now, we build the scheduling groups. The number of scheduling groups is equal to the cardinality of $P_{\mathrm{ec}}$. If no other user satisfies the above condition for user $u_{m}$, the corresponding scheduling group for user $u_{m}$ is composed of user $m$ alone. Then, user $m$ occupies a subchannel on its own in the network. To build a scheduling group for $u_{m}$, we elect an appropriate member out of $S_{m}$ for every neighbor RAU. Therefore, at most one user that is being served by a neighbor RAU belongs to the scheduling group for $u_{m}$.

Let $g_{m}$ be the scheduling group for $u_{m}$ in $P_{\mathrm{ec}}$. Then, $g_{m}$ is defined by following algorithm.

Algorithm 1 (scheduling group).

(1) Pick one user $u_{m}$ from $P_{\mathrm{ec}}$.

(2) Determine the preferred candidate set $S_{m}$ for $u_{m}$.

(3) Select a RAU $\Phi$ from the neighbor RAU of $u_{m}$

(4) Perform $g_{m} \ni \psi_{j}$, if $\psi_{j}=\arg \left\{\max _{j} c_{m, n \mid \Psi=\left\{\psi_{j}\right\}}^{\pi(m)}+c_{\psi_{j}, n \mid \Psi=\{m\}}^{\pi\left(\psi_{j}\right)}\right.$, $\psi_{i} \in S_{m}$, and $\left.\pi\left(\psi_{j}\right)=\Phi\right\}$ exists.

(5) Update $S_{m}=S_{m}-\left\{\psi_{j}\right\}$.
(6) Repeat (3) (5) for all neighbor base stations of $u_{m}$.

(7) Repeat (1) (6) for every user in $P_{\mathrm{ec}}$.

After building the scheduling groups, we calculate the capacity of each group for each subchannel.

$$
c_{g_{i}, n}=\sum_{k=1}^{n\left(g_{i}\right)} c_{k, n}^{\pi(k)} .
$$

Here, $n\left(g_{i}\right)$ is the cardinality of set $g_{i}$. Then, we allocate the subchannels for each scheduling group according to the following policy when $N_{\mathrm{ch}}$ is the number of subchannels and $n\left(P_{\mathrm{ec}}\right)$ is the cardinality of $P_{\mathrm{ec}}$ :

$$
\begin{array}{ll}
\max & \sum_{n=1}^{N_{\mathrm{ch}} n\left(P_{\mathrm{ec}}\right)} \sum_{i=1} I_{g_{i}, n} \cdot c_{g_{i}, n} \\
\text { s.t. } & \sum_{i=1}^{n\left(P_{\mathrm{ec}}\right)} I_{g_{i}, n}=1 \quad \text { for each } n .
\end{array}
$$

Note that the above optimal scheduling problem for overall network has exactly the same computational complexity as that of the simple scheduling problem for singlecell optimization [8]. This is because the unit of scheduling is expanded from a user to a scheduling group. Therefore, the complexity will not be increased compared to single-cell scheduling problem. And the scheduling algorithm can be implemented much more easily in practical systems.

\section{Performance Evaluation}

We assume that users are distributed randomly over the network area. Let $N_{i \text {,in }}$ be the number of users in the inner side of RAU $i$ in the exclusive cluster. Let $N_{i \text {,out }}$ be the number of users in the outer side of RAU $i$ in the virtual cell who do not cause severe interference to each other because they are not located in the exclusive cluster. Further, there exist $N_{r}$ RAUs in the virtual cell. Then, the total number of users is defined as $N=\sum_{i=1}^{N_{r}} N_{i, \text { in }}+N_{i \text {,out }}$.

If the network resources are utilized fully, every subchannel will be occupied by a certain user. Then, in this case, every subchannel is used by $N_{r}$ users in the entire network because at least one user occupies the subchannel in each cell. Therefore, the probability $P_{\text {inter }}$ that a user in $P_{\mathrm{ec}}$ receives strong interference in a subchannel due to a severe interferer is calculated as follows:

$$
P_{\text {inter }}=1-\frac{\prod_{i=1}^{N_{r}}\left(\begin{array}{c}
N_{r} \\
N_{i, \text { out }}
\end{array}\right)}{\prod_{i=1}^{N_{r}}\left(\begin{array}{c}
N_{r} \\
N_{i, \text { in }}+N_{i, \text { out }}
\end{array}\right)} .
$$

Figure 14 shows the variation of $P_{\text {inter }}$ according to $\sum_{i}^{N_{r}} N_{i, \text { in }}$. Here, $\sum_{i}^{N_{r}}\left(N_{i, \text { in }}+N_{i \text {,out }}\right)$ is fixed as 15 . Then, we vary $\sum_{i}^{N_{r}} N_{i, \text { in }}$ from 5 to 10 . In the case that $\sum_{i}^{N_{r}} N_{i \text {,in }}$ is 5 , the probability that the some of inner-area users receive strong interference is greater than 0.7. Moreover, in the case that $\sum_{i}^{N_{r}} N_{i, \text { in }}$ is 10 , the probability approaches 1 . However, in the proposed algorithm, this problem can be avoided. 


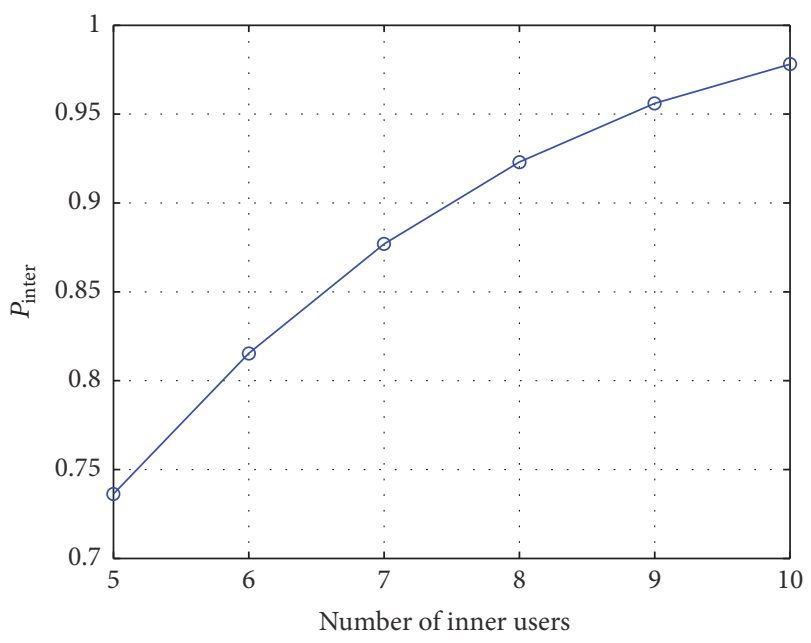

FIgURe 14: Probability that a user in an exclusive cluster receives strong interference due to a severe interferer versus number of innerarea users.

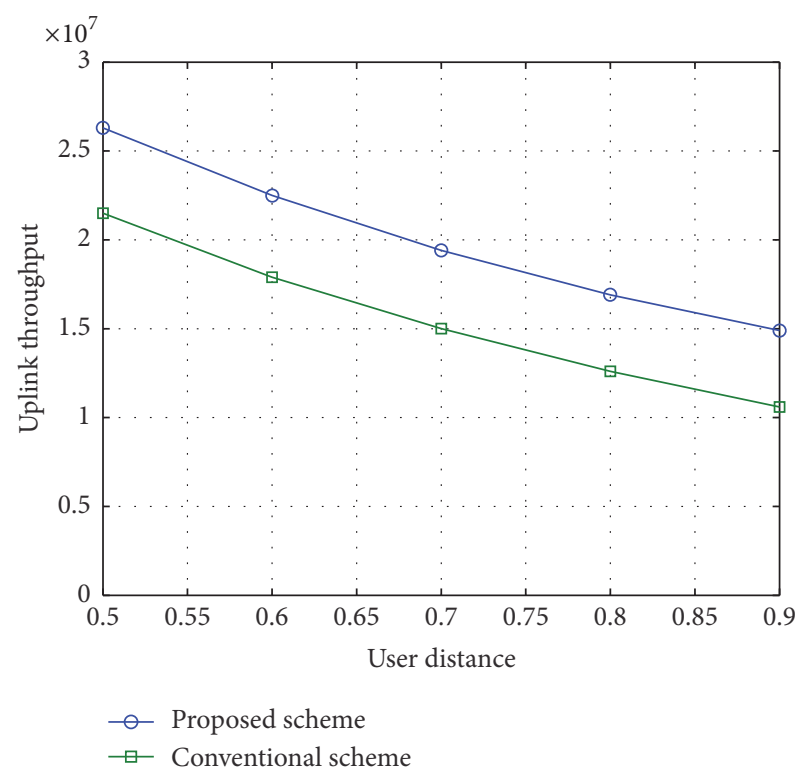

FIGURE 15: Uplink throughput versus user distance.

To analyze the performance of the proposed algorithm, we validated the proposed algorithm in a coordinated network by computer simulations using MATLAB. Then, we observed the uplink throughput in the following scenario.

We used the following parameters. The frequency was $2 \mathrm{GHz}$, the available bandwidth was $10 \mathrm{MHz}$, and the transmission power was $100 \mathrm{~mW}$, which is usual in indoor communication systems. An Okumura-Hata channel model for indoor environments was used. The number of coordinated RAUs was assumed to be 3 and five users were located randomly in the area served by each base station. The distance between RAUs was $20 \mathrm{~m}$, and the number of subchannels was 18.

Figure 15 shows the uplink throughput according to the location of the users. On the $x$-axis, the ratio of the user location to the coverage of the RAU is plotted. The proposed scheme outperforms the conventional scheme in terms of throughput. In the conventional scheme, asymmetric intercell interference cannot be taken into account because the uplink channel quality of neighbor cell users is not used for uplink scheduling. Thus, uplink traffic is scheduled by using only the information of the downlink channel that is measured from the pilot signal. In this conventional network configuration $[1,2,8]$, the performance of conventional scheme is measured by using single-cell optimization of uplink scheduling based on downlink channel information.

The uplink throughput falls as the distance of the users from the RAU increases because the path loss increases as the distance increases. In contrast, when the proposed algorithm is used, the capacity gain in uplink increases up to $50 \%$, relative to the conventional algorithm. This is because the effect of intercell interference is reduced by applying the proposed algorithm. Moreover, the asymmetric property of uplink intercell interference is intensified as the distance of the user is increased. This is because the uplink interference to neighbor cells is increased and the signal strength to the serving cell is decreased as the distance of the user is increased. Thus, the downlink channel information is more incorrect for uplink scheduling of the users in outer regions. Also, the amount of performance enhancement is relatively increased in case that the users are located in outer regions as the distance of the user is increased.

\section{Conclusion}

We have proposed an efficient intergroup scheduling algorithm for self-organizing virtual cell networks. To overcome the problem of the asymmetry in interference between downlink and uplink, we introduced scheduling groups, in which the same resource is shared by multiple users. Then, network-wise scheduling was performed to mitigate uplink interference. The numerical and simulation results show that the proposed algorithm can reduce the probability that near interferers in the network share the same resource, thereby improving uplink performance.

\section{Competing Interests}

The authors declare that there is no conflict of interests regarding the publication of this paper.

\section{References}

[1] J. Huang, V. Subramanian, R. Agrawal, and R. Berry, "Joint scheduling and resource allocation in uplink OFDM systems for broadband wireless access networks," IEEE Journal on Selected Areas in Communications, vol. 27, no. 2, pp. 226-234, 2009.

[2] A. Kumar, Y. Liu, and A. Wason, "LTE-advanced: the roadmap to $4 \mathrm{G}$ mobile wireless networks," Global Journal of Computer Science and Technology, vol. 10, no. 4, pp. 50-53, 2010.

[3] 3GPP, "Aspects of joint processing for downlink CoMP, 3GPP TSG RAN WG1,” 3GPP R1-090942, 2009.

[4] 3GPP R1-083050, Inter-eNB and Intra-eNB Schemes for CoMP in LTE-Advanced, 3GPP TSG RAN WG1, August 2008. 
[5] V. Jungnickel, K. Manolakis, W. Zirwas et al., "The role of small cells, coordinated multipoint, and massive MIMO in 5G," IEEE Communications Magazine, vol. 52, no. 5, pp. 44-51, 2014.

[6] P. K. Agyapong, M. Iwamura, D. Staehle, W. Kiess, and A. Benjebbour, "Design considerations for a $5 \mathrm{G}$ network architecture," IEEE Communications Magazine, vol. 52, no. 11, pp. 65-75, 2014.

[7] M. Rahman and H. Yanikomeroglu, "Enhancing cell-edge performance: a downlink dynamic interference avoidance scheme with inter-cell coordination," IEEE Transactions on Wireless Communications, vol. 9, no. 4, pp. 1414-1425, 2010.

[8] B. Rengarajan, A. L. Stolyar, and H. Viswanathan, "Selforganizing dynamic fractional frequency reuse on the uplink of OFDMA systems," in Proceedings of the 44th Annual Conference on Information Sciences and Systems (CISS '10), pp. 1-6, Princeton, NJ, USA, March 2010. 

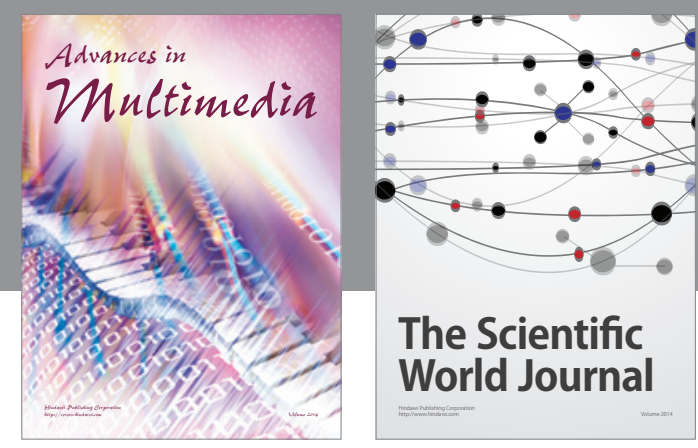

The Scientific World Journal
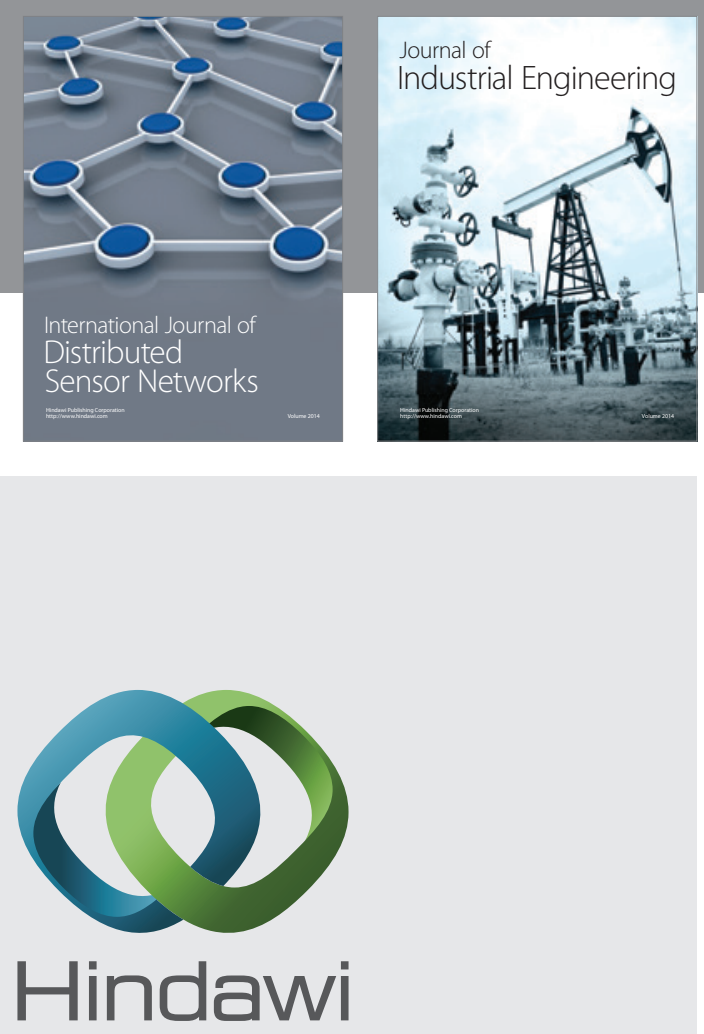

Submit your manuscripts at

http://www.hindawi.com

\section{Computer Networks} and Communications
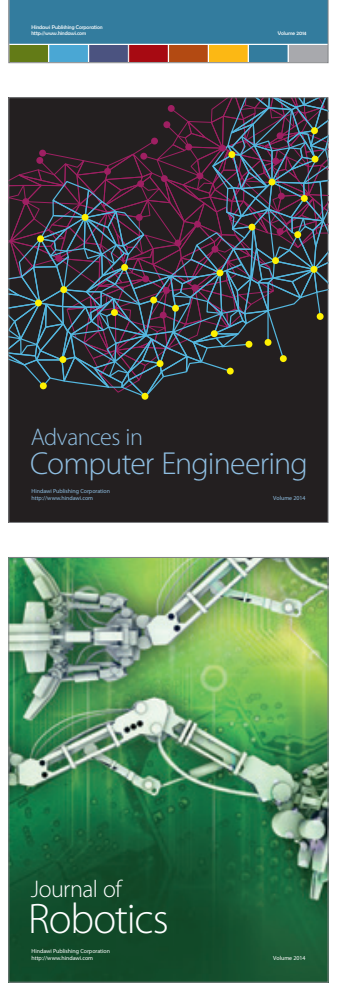
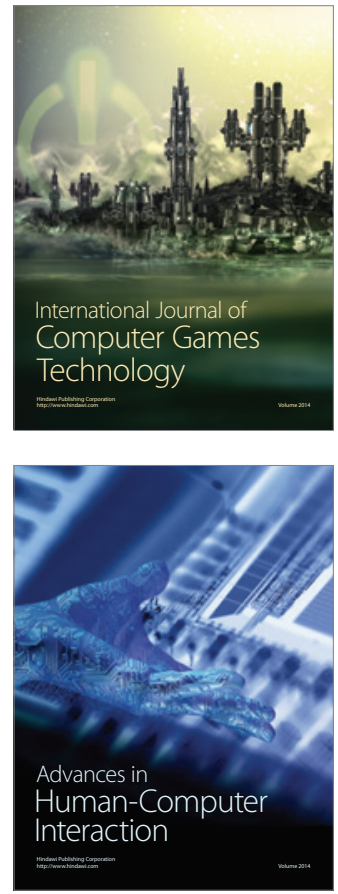
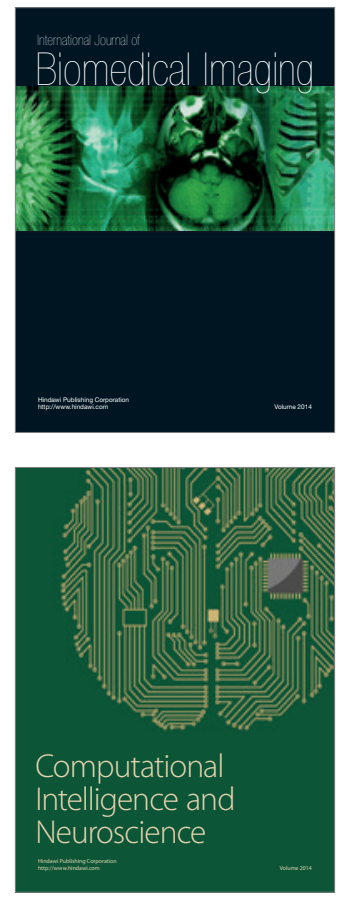
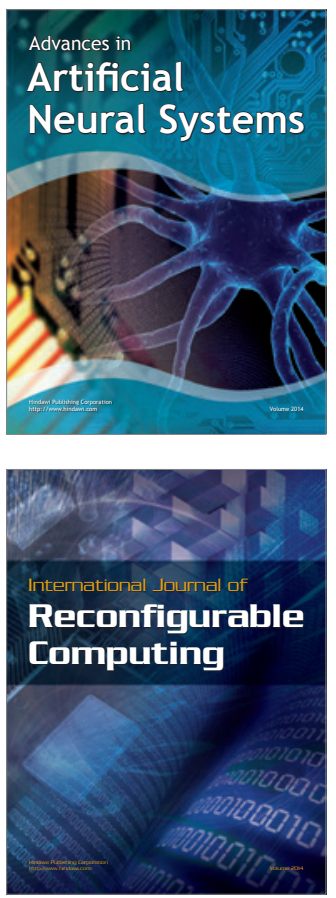
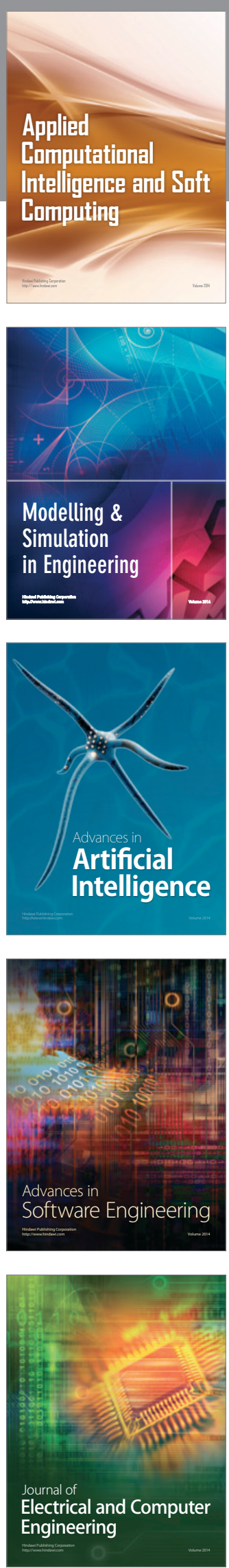